\title{
Foundation of a New Digital Ecosystem for u-Content: Needs, Definition, and Design
}

\author{
Yoosoo $\mathrm{Oh}^{1}$, Sébastien Duval ${ }^{1}, \mathrm{Sehwan} \mathrm{Kim}^{2}$, Hyoseok Yoon ${ }^{3}$, Taejin $\mathrm{Ha}^{3}$, \\ and Woontack Woo ${ }^{3}$ \\ ${ }^{1}$ GIST CTI, 261 Cheomdan-gwagiro (Oryong-dong), Buk-gu \\ Gwangju, 500-712 Republic of Korea \\ ${ }^{2}$ WorldViz, 614 Santa Barbara St, Santa Barbara, CA 93101, USA \\ ${ }^{3}$ GIST U-VR Lab., 261 Cheomdan-gwagiro (Oryong-dong), Buk-gu \\ Gwangju, 500-712 Republic of Korea \\ \{yoh, sduval\}@gist.ac.kr, kim@worldviz.com, \\ \{hyoon, tha, wwoo\} @gist.ac.kr
}

\begin{abstract}
In this paper, we analyze and classify digital ecosystems to demonstrate the need for a new digital ecosystem, oriented towards contents for ubiquitous virtual reality (U-VR), and to identify appropriate designs. First, we survey the digital ecosystems, explore their differences, identify unmet challenges, and consider their appropriateness for emerging services tightly linking real and virtual (i.e. digital) spaces. Second, we define a new type of content ecosystem (u-Content ecosystem) and describe its necessary and desirable features. Finally, the results of our analysis show that our proposed ecosystem surpasses the existing ecosystems for U-VR applications and contents.
\end{abstract}

\section{Introduction}

An ecosystem is a system defined by the interactions of a community of organisms with each other and with their environment; in a natural ecosystem, the natural environment is in which interdependent organisms, such as plants and animals, and physical factors of the environment, such as rocks and soil. [1] The interactions between the organisms in computing resources (e.g., devices, systems) including users can define non-natural ecosystems such as digital, mobile, or content ecosystems.

Many researchers describe digital ecosystems [2-5], defined as ecosystems formed by computing resources (e.g. devices, services, data), in order to acquire new perspectives on information systems. Some researchers focus on mobile ecosystems [6], and more rarely on content ecosystems. Mobile ecosystems are digital ecosystems in which the mobility of users drives interactions and evolutions. Typically, a mobile device informs a service about its position, orientation or movement, and the service processes this information to suggest or provide appropriate contents. For example, a smart phone may use an embedded GPS to tell its position to a navigation service, which calculates and displays a path to the user's destination, indicating restaurants on the way. Besides, content ecosystems are digital ecosystems that preserve the order of 
contents that are created, maintained, modified, evolved, or destroyed by means of users' participation. Typically, the devices provide stable standard hooks (e.g. API, codec), and are considered more or less equivalent from a systems perspective; rather than organisms, they become parts of the environment (which does not preclude improvements).

Mobile ecosystems and content ecosystems can both be extended but are basically disjoint; their features can promote services in ubiquitous virtual reality but are difficult to combine in their current form. Consequently, it is important to define and realize a new type of ecosystem for u-Contents (u-Content ecosystem), based on mobile and content ecosystems, ideally with minimal fundamental changes to effectively and efficiently reuse existing works. Here, we use u-Contents according to the following definition: "contents appropriate for an UbiComp-enabled smart environment, making virtual reality pervasive into our daily lives" [7]. The u-Content highlights the importance of users, who are continuously immersed in a mix of real and virtual spaces (aka U-VR [8] space), whose contexts and activities influence the relevance of contents and services, and who may produce as much as consume contents.

For the u-Content ecosystem, we should precisely match contents to users' characteristics (e.g. abilities, preferences, viewpoints), to the physical world (e.g. location, luminance, noise), events, social situations, and to goals of producers and consumers. We also need to motivate and support a sustainable participation of users as mobile producers and consumers, which implies simple contents creation, flexible distribution, easy retrieval, and seamless interaction. Finally, the permanent selfgrowth of contents is highly desirable.

Through this paper, we consolidate information from diverse sources, providing a consistent bibliography from fields such as information systems, human-computer interaction, and the semantic web. Our survey revealed 8 key concepts: digital food (resource needed by contents), digital organ (component of contents), digital species (type of contents on various platforms and devices), digital community (group of contents), digital environment (e.g. mobile device, intranet, Internet), digital ecosystem type (e.g., mobile, contents, digital health ecosystems), eco-relationship (relationship between two or more digital species), and eco-law (e.g. format, application specification and restriction). Based on our thorough analysis using the key concepts, our taxonomy helps visualize all related aspects and find out essential technical components for u-Content ecosystems.

Moreover, we demonstrate that traditional digital ecosystems cannot appropriately process u-Contents due to low considerations for human factors (e.g. restrictions for kids, adaptations for novices, rewards to motivate end-user participation), energy management (e.g. contents download consumption), and users' context (e.g. profile, user-created contents, location, time, activity, belonging to a community). Our analysis also highlights weaknesses in the temporal management (up-to-date/realtime, old/archived, future/predictions), in the contents management (equal versus unequal treatment), and in the provision of alternatives to find, combine (e.g. subtitles, translations), transform (e.g. format, version), and use contents.

In this paper, we propose the foundation of a new digital ecosystem for u-Content. First, we survey the digital ecosystems, explore their differences, identify unmet challenges, and consider their appropriateness for emerging services tightly linking 
real and virtual (i.e. digital) spaces. Second, we define a new type of content ecosystem (u-Content ecosystem) and describe its necessary and desirable features.

The proposed u-Content ecosystem enables new services while facilitating the realization-and enhancing the quality-of existing services. Elements of interest concerning our $\mathrm{u}$-Content ecosystem notably include the provision of adaptation mechanisms to enable personalization and situational focus, and of reward mechanisms for content contributors to encourage participation. Finally, the results of our analysis show that our proposed ecosystem surpasses the existing ecosystems for U-VR applications and contents.

\section{Key Concepts and Analysis}

In this section, we define, clarify and exemplify the 8 key concepts that our survey revealed: digital food, digital organ, digital species, digital community, digital environment, digital ecosystem, eco-relationship, and eco-law. We then analyze related literatures by applying the key concepts and sometimes we interpret the literatures based on our view.

\subsection{The 8 Key Concepts of the Digital Ecosystem}

Digital foods are resources individuals must consume to survive and prosper [3], including data, memory space, and processing cycles. Here, "consume" means various things: data may remain available without change, memory may be freed later, and processing cycles may be replenished.

Digital organs are permanent software and hardware parts of individuals that support specific features of digital species [2]. Mobile ecosystems typically feature antennas and algorithms for power management, localization and face recognition; content ecosystems notably feature affective meta-data [9].

Digital species mimic biological species, living organisms that are autonomous, viable, and self-organizing, based on purposes, hardware and software [2, 3]. Alternatively, some consider the abstractions of the devices as species, instead of the devices themselves [3]. In any case, digital species may be neutral to, benefit or harm their ecosystem. In generic ecosystems, digital species notably include computers, displays, cameras, smart cards, and card readers; in specific ecosystems such as health ecosystems, they may be medical devices or measuring tools [2]. In content ecosystems, digital species notably include 3D models and 3D contents.

Digital communities are groups formed by digital species, mimicking communities of intelligent or stupid animals and plants [2].

Digital environments are environments in which digital species evolve, and in which individuals complete their life-cycle. Environments apply pressure but also offer cradles, dwellings, graves, and resources (e.g. information and transaction flows [2]). Digital environments may be spatial [3], with functions to indicate local resources and neighbors, or not; for example, environments for virtual reality are typically spatial whereas the World Wide Web is basically non-spatial. Ecological niches associated to idiosyncratic eco-laws may form the environment [3], with a dynamic hierarchy of niches containing one or more niches (enclosing niches) and 
fully contained niches (enclosed niches). In generic ecosystems, nested ecological niches may be printers in smart rooms in smart buildings in a smart city. In health ecosystems, information includes personal health records, and transactions include verifications and money transfers when purchasing drugs [2].

A digital ecosystem is a "dynamic and synergetic complex of digital communities consisting of interconnected, interrelated, and interdependent digital species situated in a digital environment that interact as a functional unit and are linked together through actions, information, and transaction flows" [2]. Examples include content ecosystems, mobile ecosystems, pervasive ecosystems [3], health ecosystems [2], and cyber-physical ecosystems such as the Energy Web [10].

Eco-relationships are relationships between digital species such as commensalism, mutualism, parasitism, and predation. Although central in Nature, predation is rarely presented in digital ecosystems; for examples of digital predation, see [3]. Hadzic and Chang [2] defines digital symbiosis as similar to biological symbiosis, with 3 types: mutualism (both species benefit), commensalism (one species benefit with no cost to the other) and parasitism (one species benefits at cost to the other). For example, digital symbiosis can appear between decision-support and reservation systems [2].

Eco-laws are fixed permanent laws regulating local interactions and changes in a digital ecosystem $[3,10]$. They may determine how and when individuals can reproduce, find/affect/kill individuals, or produce/find/alter/consume resources [3]. Villalba et al. [3] distinguishes internal from external laws, managing what happens respectively within a niche and between a niche and the rest of the space. Elsewhere, the laws may be phylogenetic (i.e. linked to the evolution of species), ontogenetic (i.e. linked to individual development, with little environmental influence) or epigenetic (i.e. linked to learning from environmental interactions) [10].

Finally, humans and communities thereof strangely fit digital ecosystems: people can be seen on a par with digital species or environmental resources... or may be ignored altogether. When considered, their main generic categories are producer [3] (e.g. designer, advertiser [11], service developer [3]), consumer [3, 10] (e.g. audience [11]), prosumer [10], and maintainer/owner (e.g. display owner [11]).

\subsection{Analysis of the Survey}

One of examples for digital foods is museum contents which include objects like images and their metadata for tagging and annotating [12]. That is, different groups of stakeholders (museum curators, owners, professional researchers and the general public) add new objects and attributes, edit, and browse the collection individually. Another example of digital foods is contents/articles which are posted on a web-page (i.e. Slashdot.org) for idea sharing. Slashdot is a virtual community site where community members write articles. These articles usually comment on current affairs, such as a report or news item written elsewhere, which the author then critically summarizes and links to [13]. Liu et al. [14] proposed designed and implemented a robust and adaptive super-peer based algorithm to construct and manage the digital ecosystem where each digital device (node) sends service requests to share resources. We can consider these kinds of service requests and services themselves as digital foods. Hadzic and Chang [2] defined digital organs as software and hardware that will support the digital species features. 
Boley and Chang [15] defined agents as entities that join an environment or a community based on their own interests, and species as types of agents, respectively. Hadzic and Chang [2] described digital organs are assembled into a digital species body. They mentioned the majority of digital species consist of 'hardware' together with its associated 'software'. Briscoe [16] mentioned that the agents of the digital ecosystem are functionally parallel to the organisms of biological ecosystems, including the behavior of migration and the ability to be evolved. In [12], we can think of various management interfaces, such as the tagging and browsing software and associated hardware, as digital species. That is, they provide different user interfaces for adding/editing new objects and attributes, and for browsing/searching the collection. It seems that Wagner et al. exemplified digital species as a unique online comment moderation process engine that is "slash" engine. Anyone can comment, even anonymously, but comments are subsequently rated for quality for balancing egalitarian forces with quality assurance [13]. In [14], a large collection of digital devices and super-peer based algorithm to construct and manage the digital ecosystem overlay could be digital species. Digital devices communicate through message exchanges, and every digital device is a node in the digital ecosystem overlay.

Even though Boley and Chang [15] mentioned the concept of digital communities, those are very similar to the description of a digital environment. Hadzic and Chang [2] also considered digital communities as some groups in a digital environment. Briscoe [16] simply defined a digital community as strongly connected cluster or group based on [17] where Begon et al. defined a community as a naturally occurring group of populations from different species that live together, and interact as a selfcontained unit in the same habitat which is a distinct part of the environment. In the virtual museum application [12], digital community/environment can be considered development platform relying on web technologies (including web 2.0 and web services) and semantic web technologies.

Boley and Chang [15] defined an (agent) environment as an environment which contains human individuals, information services as well as network interaction and knowledge sharing tools along with resources that help maintain synergy among human beings or organizations. The definition of a digital environment by Hadzic and Chang [2] is an environment in which digital species jointly live, function, and relate. In addition, they mentioned that the digital environment is very analogous to the biological environment, and the digital environment was considered as an important constituent for digital ecosystem. Briscoe [16] considered an environment as population's habitat where different species live together and interact.

Digital ecosystems are self-organizing systems which can form different architectural models through swarm intelligence, where local interactions between agents determine the global behavior [15]. Hadzic and Chang [2] defined a digital ecosystem as the dynamic and synergetic complex of digital communities consisting of interconnected, interrelated, and interdependent digital species situated in a digital environment that interact as a functional unit and are linked together through actions, information, and transaction flows. Briscoe [16] considered digital ecosystems software systems that exploit the properties of biological ecosystems. Eklund et al. [12] designed a digital ecosystem of the virtual museum of the pacific, which is a web-based knowledge management system (or information space) for ethnographic 
objects. Wagner et al. [13] defined a digital ecosystem as a kind of community where different parties with different levels of interest and resources participate and provide resources in a give-and-take that creates benefits for all. A digital ecosystem is an emerging paradigm for economic and technological innovation to support the cooperation, knowledge sharing and development of open and adaptive technologies [18].

It seems that Boley and Chang [15] emphasized the benefit and profit of species through the relationship within communities. Precisely, collaboration between entire digital ecosystems should be able to provide benefits to all ecosystems involved. In addition, participation of individuals in multiple digital ecosystems helps them to reach their goals. Eklund et al. described relationships across ecosystems as any inferences that are formed as a result of user communities tagging and annotating objects (or writing wiki entries) in their own group [12]. For example, historians, anthropologists and curators are each members of their own 'group'. If anthropologists are a group within their own 'ecosystem' and the historians belong to another, a cross-ecosystem relationship is formed from their respective collaborative inputs into the virtual museum. Wagner et al. [13] stressed on a dynamic give-andtake between community members to achieve benefits for all through an effective information and coordination mechanism, those also require little learning and little effort to maintain, i.e., a well designed system enabling the professional virtual community. Sometimes, species cooperate with each other in order to share resources [14]. They dispatch the service requests from users to client nodes. One peer group is able to provide a type of service that differs from the other peer groups.

An example of eco-law could be the acceptance and rejection process by moderating editors [13]. After articles are posted by community members on the virtual community site, there is a moderation process for each article.

\section{Ecosystem Realizations}

To realize ecosystems, we may integrate many complementary concepts and technologies.

\subsection{Necessary and Desirable Features}

In the case of content ecosystems, the Semantic Web techniques (based on formal syntaxes, ontologies, inference rules) can greatly support human-machine and machine-machine interoperability. For example, we can consider discovery process based on semantic matching within a niche (or nearby one) [3]. Besides, Villalba et al. [3] identifies a single key component for passive species: a public semantic description of its features; active species add: a set of needs e.g. nature of its foods, a happiness status driving activities, and an internal logic to maximize its happiness. Here, happiness is typically goal/need-oriented and time-dependent, and may depend on the happiness of nearby individuals. These components may notably enable evolvability and self-healing but may hamper privacy awareness and self-protection. Finally, individuals/species should ultimately be endowed with manipulation to 
reconfigure themselves and the (real) world/environment [19]. This ability would most notably increase robustness and self-protection while uniquely helping deal with limited access.

\subsection{Application}

This section exposes the scope and interest of attempts to establish ecosystems so far and in particular content ecosystems. The concept of ecosystem has been applied to the domains of advertisement, energy and health. Besides, Hadzic and Chang [2] suggested digital business ecosystems, digital government ecosystems, digital law ecosystems, and digital industry ecosystems but without clarifying their potential components.

In the domain of advertisement, Villalba et al. [3] discussed an advertising ecosystem. For example, in MyAds [11], information and advertising agents could move among screens, looking for people with specific user profiles, and then try to control a selected screen with their own contents [3]. In the "display niches", information feeds user agents, who feed advertiser agents then display-bound monitoring agents selectively spread information and guide advertiser agents, forming a feedback loop; eco-laws define how user agents make user profiles available in the displays [3]. In cloud-based ad printing service [20], web content is automatically reproduced with automatic contextual ad and coupon insertion when the web content is printed.

In an Energy Web system [10], autonomous agents control the production and usage of distributed renewable energy sources to optimize energy use and allow rapid innovation. It is envisioned based on 5 layers: distribution, control, prosumer, community, and incentive (from more technical to more social). A digital health ecosystem has been proposed, focusing on electronic health records, notably involving hospitals, health services, general practitioners, pharmacies, health systems, and health information resources [2]. According to [19], ecosystems may increase the efficiency of, and innovation in, mHealth (e.g. disease prevention and management) by providing flexibility.

In the domain of $\mathrm{P} 2 \mathrm{P}$ applications, the BitTorrent ecosystem is one of the successful open internet applications in the present. Zhang et al. [21] crawled popular torrent-discovery sites than studied in-depth the Ecosystem's tracker, peer, user behavior, and content landscapes. In the domain of digital museums, information and knowledge of museum artifacts have been digitally collected to construct the ecosystem of the virtual museum [12]. Additionally it encourages sustainable user participant by containing a wiki component, where the ecosystem enables the creation of user-defined perspectives with semantic theme.

All these ecosystems could be merged into a universal digital ecosystem [2], in the same sense that our natural ecosystems can be merged into an Earth ecosystem. Conversely, many digital ecosystems could be split into lower-level ecosystems; for example, a digital ecosystem may be split into a digital dental ecosystem, a digital physiotherapy ecosystem, a digital surgery ecosystem, and so on [2]. 


\section{Discussion}

Considering u-Content and its characteristics, the existing ecosystems have strong limitations linked to contents manipulation, privacy, standards, user participation, and management.

Contents are still difficult to manipulate, notably because of structures (e.g. file formats) are not organized to allow innovations and because semantic information is missing. However, solutions can be integrated in ecosystems. For example, to facilitate pervasive video editing, Paluska et al. [22] proposed the concept of chunk: a "typed, ordered, fixed-size array of fixed-size slots" with slots holding scalar data and linking to another chunk. ChunkStream demonstrated adaptations to local constraints [22]: bandwidth using a search tree, and screen sizes using several fidelity levels. These chunks can be generalized to other types of applications and contents. Regarding semantic information, many types of contents lack semantics, techniques to provide semantics, and tools to enable interoperability; for instance 3D media [23], sounds and smells. For u-Content manipulation within the ecosystem, representations of u-Reality, u-Intelligence, and u-Mobility need to be formally defined. Metadata that describe u-Content can be further utilized in means of context-aware manipulation for filtering and recommendation. With this support, $\mathrm{u}$-Content can be updated and modified by multiple users and enriched collectively by users as well as between interactions of u-Contents.

u-Content may be particularly constrained legally because, by their nature, they heighten privacy threats. For example, Europe's rights commissioner discussed in 2010 a legal "right to be forgotten" online (correction, withdrawal, deletion of contents) that may be included in a soon-to-come update of the EU data protection rules [24]. Existing ecosystems do not particularly facilitate contents removal. Ecosystem features that regulate what to share, how to share, who gets the right/ownership and granting accessibility of who can view, edit and publish need to be included in u-Content ecosystem.

Ecosystems typically lack standardized formats and (high capacity, quality) infrastructures to share contents, which is particularly true for health services [2]. This is also true for $\mathrm{u}$-Content but the difference is that $\mathrm{u}$-Contents are just emerging: now is the best moment to establish standards, now that we can see what u-Content should be, and before specific solutions become de facto standards. Such activities are currently taking place in u-Content [7] and mixed reality content representation [25].

User participation is an important feature missing in usual digital ecosystems. A user may stop providing contents or feedback if her costs and expectations exceed her benefits. For participatory sensing, costs include bandwidth, CPU and energy consumption as well as privacy [8]. For user participation, Lee et al. [8] proposed a Reverse Auction based Dynamic Price incentive mechanism with Virtual Participation Credit, maintaining a sufficient number of users providing environmental data while lowering costs to purchasers. Provision of incentives to users as well as providing gaming and engaging experiences should also be included in u-Content ecosystem.

Finally, we lack efficient techniques to manage distributed online evolution [10], suitable ways to measure fitness in a distributed way with noise [10], suitable metrics to assess robustness and resilience in autonomic complex systems [10], guidance for 
diagnosing e.g. simulations to test ecosystems and security assessments. Villalba et al. [3] even points out that we ignore what the threats are in a digital ecosystem.

Considering the properties we wish to imbue, and the limitations noted so far, it appears that inventing one or several appropriate frameworks will require direct hands-on experience.

\section{Conclusion}

In this paper, we analyzed digital ecosystems to demonstrate the need for a new digital ecosystem, oriented towards contents for ubiquitous virtual reality. We surveyed the digital ecosystems and defined a new type of content ecosystem (uContent ecosystem). Through this paper, we consolidated information from diverse sources, providing a consistent bibliography from fields such as information systems, human-computer interaction, and the semantic web. Based on the results of our analysis, we showed that our proposed ecosystem surpasses the existing ecosystems for U-VR applications and contents. We expect our proposal to lead to self-emergence at both the micro (better contents and more interesting links between contents) and macro (prosumers) levels.

\section{References}

1. Wikipedia, http://en.wikipedia.org/wiki/Ecosystem

2. Hadzic, M., Chang, E.: Application of Digital Ecosystem Design Methodology Within the Health Domain. IEEE Transactions on Systems, Man, and Cybernetics-Part A: Systems and Humans 40(4), 779-788 (2010)

3. Villalba, C., Mamei, M., Zambonelli, F.: A self-organizing architecture for pervasive ecosystems. In: Weyns, D., Malek, S., de Lemos, R., Andersson, J. (eds.) SOAR 2009. LNCS, vol. 6090, pp. 275-300. Springer, Heidelberg (2010)

4. Dong, H., Hussain, F.K., Chang, E.: Semantic Service Retrieval and QoS Measurement in the Digital Ecosystem Environment. In: International Conference on Complex, Intelligent and Software Intensive Systems (CISIS), pp. 153-160 (2010)

5. Pfeifer, T., Malone, P.: Digital Ecosystem for Cross-domain Context Sensing and Tradinz. In: 32nd IEEE Conference on Local Computer Networks (LCN 2007), pp. 228-230 (2007)

6. Xia, R., Rost, M., Holmquist, L.E.: Business Models in the Mobile Ecosystem. In: 2010 Nineth International Conference on Mobile Business / 2010 Ninth Global Mobility Roundtable, pp. 1-8 (2010)

7. Kim, K., Han, J., Kang, C., Woo, W.: Visualization and Management of u-Contents for Ubiquitous VR. In: The 14th International Conference on Human-Computer Interaction (2011) (to be published)

8. Lee, Y., Oh, S., Shin, C., Woo, W.: Ubiquitous Virtual Reality and Its Key Dimension. In: International Workshop on Ubiquitous Virtual Reality, pp. 5-8 (2009)

9. Tkalčič, M., Burnik, U., Košir, A.: Using Affective Parameters in a Content-based Recommender System for Images. User Modeling and User-Adapted Interaction 20(4), 279-311 (2010)

10. Carreras, I., Miorandi, D., Saint-Paul, R., Chlamtac, I.: Bottom-Up Design Patterns and the Energy Web. IEEE Transactions on Systems, Man, and Cybernetics-Part A: Systems and Humans 40(4), 815-824 (2010) 
11. Di Ferdinando, A., Rosi, A., Lent, R., Manzalini, A., Zambonelli, F.: MyAds: A System for Adaptive Pervasive Advertisements. Pervasive and Mobile Computing 5(5), 385-401 (2009)

12. Eklund, P., Wray, T., Goodall, P., Bunt, B., Lawson, A., Christidis, L., Daniels, V., Van Olffen, M.: Designing the Digital Ecosystem of the Virtual Museum of the Pacific. In: 3rd IEEE International Conference on Digital Ecosystems and Technologies, pp. 805-811 (2009)

13. Wagner, C., Liu, L., Schneider, C., Prasarnphanich, P., Chen, Huaping.: Creating a Successful Professional Virtual Community: A Sustainable Digital Ecosystem for Idea Sharing. In: Digital Ecosystems and Technologies (DEST 2009), pp. 163-167 (2009)

14. Liu, M., Koskela, T., Ylianttila, M.: Constructing a Robust Overlay for Digital Ecosystems. In: Digital Ecosystems and Technologies (DEST 2009), pp. 430-435 (2009)

15. Boley, H., Chang, E.: Digital Ecosystems: Principles and Semantics. In: Digital Ecosystems and Technologies (DEST 2007), pp. 398-403 (2007)

16. Briscoe, G.: Creating a Digital Ecosystem: Service-Oriented Architectures with Distributed Evolutionary Computing. In: Java One Conf., p. 0759 (2006)

17. Begon, M., Harper, J., Townsend, C.: Ecology: Individuals, Populations and Communities. Blackwell Publishing, Malden (1996)

18. Nachira, F., Dini, P., Nicolai, A., Le Louarn, M., Rivera Lèon, L.: Digital Business Ecosystems. The European Commission (2007)

19. Estrin, D., Sim, I.: Open mHealth Architecture: An Engine for Health Care Innovation. Science 330(6005), 759-760 (2010)

20. Yang, S., Jin, J., Parag, J., Liu, S.: Contextual advertising for web article printing. In: The 10th ACM Symposium on Document Engineering (DocEng 2010), pp. 195-198 (2010)

21. Zhang, C., Dunghel, P., Wu, D., Ross, K.W.: Unraveling the BitTorrent Ecosystem. IEEE Transactions on Parallel and Distributed Systems (2010); Online published (10.1109/TPDS.2010.123)

22. Paluska, J., Pham, H., Ward, S.: ChunkStream: Interactive Streaming of Structured Data. Pervasive and Mobile Computing 6(6), 607-622 (2010)

23. Spagnuolo, M., Falcidieno, B.: 3D Media and the Semantic Web. IEEE Intelligent Systems 24(2), 90-96 (2009)

24. Waterfield, B.: Right to be forgotten. The Telegraph (2011)

25. Kim, H., Yoon, H., Choi, A., Baek, W., Lee, I., Kim, D., Woo, W.: Data Markup Representation for Mixed Reality Contents. In: International AR Standards Meeting 2011 (2011) 